\title{
Consumo de alcohol y tabaco entre los alumnos de un centro de formación de Fuerzas y Cuerpos de Seguridad del Estado
}

\author{
Morales Bonilla JA. ${ }^{1}$, Arandojo Morales MI. ${ }^{2}$, Garcia Cuesta J. ${ }^{1}$, Orbañanos Peiro L. ${ }^{3}$ \\ Sanid. mil. 2014; 70 (4): 248-255; ISSN: 1887-8571
}

\begin{abstract}
RESUMEN
El tabaco y el alcohol son drogas de uso legal con una amplia aceptación social y suponen el inicio en otras drogodependencias. Está probada la relación de ambas con la aparición de varias enfermedades y su consumo es cada vez mayor a edades más tempranas. Objetivos: ofrecer una visión descriptiva del consumo de drogas legales en futuros Agentes del Orden Público. Material y método: se administró a 153 alumnos una encuesta de 47 preguntas de tipo cerrado. Los análisis de las variables se realizaron mediante tabla de cálculo de Excel. Resultados: de una muestra final de 141 encuestados (91'5\% hombres y 8'5\% mujeres) de entre 18 y 24 años, hay un $37^{\prime} 6 \%$ de fumadores que han comenzado a fumar a los 13-16 años y consumen de 1 a 7 cigarrillos (74'55\%). El 98\% sabe que el consumo es cancerígeno, el $96 \%$ que es adictivo y al 18'44\% le ha fallecido algún familiar por el tabaco pero el 60\% siguen fumando para relajarse. En cuanto al alcohol, beben el $82 \%$, sobre todo cerveza (46\%). Se han iniciado en el consumo a los $13-16$ años por influencia de amigos (80'5\%) o familiares (6'5\%). Beben sobre todo los fines de semana (49'5\%) y el $81^{\prime} 3 \%$ bebe más cuando se reúne con amigos. E1 98\% sabe que produce daño hepático, el 95'5\% conocen sus efectos adictivos y el 23\% tienen familiares con problemas por el alcohol, pero el $81 \%$ se ha emborrachado alguna vez. Conclusiones: los hábitos de consumo de alcohol y tabaco en este colectivo son similares a los de la población general.
\end{abstract}

PALABRAS CLAVE: tabaco, alcoholismo, trastornos relacionados con el alcohol, hábito de fumar.

Consumption of alcohol and tobacco among students from a center of training of forces and state security bodies

SUMMARY: tobacco and alcohol are legal drugs with broad social acceptance and they are the beginning of other drug addictions. The relationship of both with the emergence of various diseases is proven and its consumption is increasing at younger ages. Objectives: provide a descriptive overview of legal drugs consumption in future Law Enforcement Officers. Material and method: a survey of 47 closed questions was administered to 153 students. The analysis of the variables was performed using Excel spreadsheet table. Results: from a final sample of surveyed 141 (91'5\% men and 8'5\% women) aged $18-24$ years, 37'6\% are smokers who have started smoking at age 13-16 and consume 1 to 7 cigarettes (74'55\%). 98\% know that consumption is carcinogenic, 96\% which is addictive and $18^{\prime} 44 \%$ have relatives who died due to tobacco, but $60 \%$ keep smoking to relax. As for alcohol, $82 \%$ drink, mostly beer (46\%). They have begun in consumption at age $13-16$ influence of friends $\left(80^{\prime} 5 \%\right)$ or family $\left(6^{\prime} 5 \%\right)$. They drink mostly weekends $\left(49^{\prime} 5 \%\right)$ and $81^{\prime} 3 \%$ drink more when they meet friends. $98 \%$ know it causes liver damage, the 95 ' $5 \%$ know their addictive effects and $23 \%$ have family troubled by alcohol, but $81 \%$ has drunk ever. Conclusions: alcohol and smoking habits of this group are similar to those of general population.

KEYWORDS: tobacco, alcoholism, alcohol-related disorders, smoking.

\section{INTRODUCCIÓN}

El tabaco y el alcohol son drogas de uso legal, de amplia aceptación social. Además, el consumo de alcohol aparece claramente relacionado con el de bebidas alcohólicas y ambas son sustancias de entrada al consumo de otras drogas, especialmente el cannabis en un segundo paso y luego al resto de drogas ilegales ${ }^{1}$.

\footnotetext{
${ }^{1}$ Cap. Enfermero.

${ }^{2}$ Cap. Enfermera

Colegio de Guardias Jóvenes "Duque de Ahumada" de la Guardia Civil. Valdemoro, Madrid. España.

${ }^{3}$ Cte. Enfermero. Escuela Militar de Sanidad. Madrid. España.
}

Dirección para correspondencia: M. ${ }^{\mathrm{a}}$ Isabel Arandojo Morales. Colegio de Guardias Jóvenes "Duque de Ahumada". Servicio Médico. Carretera de Andalucía Km 25. 28340 Valdemoro (Madrid).

Tfn: 915146820 / 6000 Ext: 45165, 45166, 45163. maribel_a@ya.com

Recibido: 13 de septiembre de 2013

Aceptado: 21 de enero de 2014
El tabaco, debido a sus efectos nocivos sobre la salud, ha sido reconocido por la OMS como la causa principal de muerte evitable ${ }^{2}$. La mayor parte de estas muertes ocurre en países de ingresos bajos y medianos y, de no evitarlos, en los próximos decenios aumentará la diferencia de mortalidad con respecto a los países de ingresos altos. Además, el tabaco y el alcohol, así como la asociación de ambos, que tiene efecto multiplicador, ocupan un lugar privilegiado entre las causas de aparición de ciertos tipos de cáncer ${ }^{3}$.

En la lucha contra el tabaquismo y el alcoholismo, es importante una acción política global como la que se propone la Organización Panamericana de la Salud ${ }^{4}$, además de centrarse en su prevención a nivel de los centros de formación en la Educación Obligatoria, debido a que el consumo de ambas sustancias se inicia en la infancia y se arraiga y consolida en la adolescencia ${ }^{1}$.

En España, según los últimos estudios publicados, las drogas de mayor consumo en la población comprendida entre $15 \mathrm{y}$ 64 años son el tabaco y el alcohol, situándose el tabaquismo en torno al 30\% y el alcohol en el 70\% según la Encuesta Nacional 
de Salud de España 2006 (ENSE 2006). En otros estudios, como la Encuesta Domiciliaria sobre Alcohol y Drogas en España 2009-2010 (EDADES 2009-2010) ${ }^{6}$, la prevalencia de 42,8\% en el consumo de tabaco y 78,7\% en el consumo de alcohol. Además, el alcohol está presente en el 90\% del policonsumo de drogas?

Hasta 2010, a pesar de los esfuerzos realizados por las administraciones responsables en materia de salud y de educación para disminuir su consumo, el problema entre la población no había disminuido $^{7}$. La aprobación de la Ley antitabaco 42/2010 de 30 de diciembre supuso un nuevo arma para combatir y reducir el consumo, y así se ha logrado una reducción de 600.000 fumadores $^{8}$.

Con respecto al alcohol, es la droga de mayor consumo en España ${ }^{7}$ debido al aumento de consumidores (adolescentes sobre todo). Según la OMS en el mundo es el causante de 2.5 millones de muertes cada año, entre los que se encuentran 320.000 jóvenes de entre 15 y 29 años y está relacionado con muchos problemas graves de índole social y del desarrollo, especialmente la violencia, el descuido, el maltrato de menores y el absentismo laboral ${ }^{9}$. Las consecuencias sobre el desarrollo biológico, psicológico y social en edades tempranas pueden ser especialmente dramáticas e irreversibles ${ }^{10}$.

Por lo tanto, el consumo de alcohol supone un problema de salud pública y ha contribuido al aumento de la morbimortalidad en numerosas enfermedades, accidentes laborales y de tráfico. La asociación entre las lesiones traumáticas y el alcohol determina la predisposición de los pacientes consumidores de alcohol a sufrir lesiones que pueden ocasionar discapacidades severas $^{11}$. Las enfermedades relacionadas con el alcohol son tanto orgánicas (digestivas, hepáticas, cardiovasculares, y neoplásicas, entre otras), como psiquiátricas (dependencias de otras sustancias, depresión, ansiedad, insomnio, violencia...) lo que puede ocasionar otros problemas sociales ${ }^{12}$.

En la siniestralidad relacionada con el alcohol, cuatro de cada diez fallecidos en accidentes de tráfico en 2010 habían consumido alcohol ${ }^{13}$, mientras que en la laboral es difícil de cuantificar, ya que esta causa no aparece o lo hace en un segundo plano, pero aun así, la incidencia se considera alta ${ }^{14}$. La Organización Internacional del Trabajo considera que el alcohol es la causa de un tercio de los accidentes laborales mortales, y que su consumo aumenta de dos a tres veces la tasa de accidentes laborales ${ }^{15}$.

\section{Objetivos}

General: describir la prevalencia del consumo de Drogas Legales (alcohol y tabaco) entre los alumnos de un centro de formación de las Fuerzas y Cuerpos de Seguridad del Estado

Especifico: comprobar el conocimiento que tienen sobre de los efectos de este consumo para poner en marcha las medidas preventivas oportunas.

\section{MATERIAL Y MÉTODO}

Estudio descriptivo transversal, con un muestreo no probabilístico de conveniencia, realizado durante el mes de enero de 2012 sobre una población diana de alumnos de un centro de formación de Cuerpos y Fuerzas de Seguridad del Estado. No se han considerado criterios de exclusión, siendo el único criterio de inclusión su voluntariedad de participar en el estudio.

Se elaboró un cuestionario autocumplimentado de 47 preguntas de tipo cerrado, sobre hábitos de consumo de tabaco y alcohol y sobre la información que tienen de las consecuencias derivadas del mismo (Tabla 1). Debido que los alumnos tienen diferente nivel de estudios, desde graduados en ESO hasta universitarios, el cuestionario se realizó utilizando un lenguaje muy coloquial para asegurarnos la comprensión del conjunto.

La encuesta se repartió a todos los alumnos reunidos en un aula, 153 individuos, y, tras explicar los objetivos del estudio y su carácter voluntario, se les dio la posibilidad de entregarla en blanco. Un investigador leyó en voz alta todas las preguntas para aclarar las posibles dudas y posteriormente se ausentó. Una vez finalizadas las encuestas, las recogieron los delegados de clase y se las entregaron al investigador.

De todas las encuestas recogidas, se rechazaron 8 por no especificar la edad o el sexo y 4 por estar en blanco, por lo que se obtuvo una muestra final de 141 individuos.

Para el análisis de los resultados se ha utilizado una tabla realizada con Microsoft Office Excel 2010, donde se han calculado porcentajes por interesarnos sólo las prevalencias.

Tabla 1. Encuesta sobre hábitos de consumo de tabaco y alcohol.

\section{SEXO \\ MASCULINO FEMENINO}

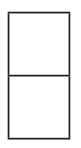

EDAD

1. ¿Fumas?
$\mathrm{Si}$
No

2. Si no lo haces, ¿Has fumado en alguna ocasión?

$$
\mathrm{Si}
$$

No
3. ¿Por qué razón no has fumado nunca?

Porque sé que causa daño

Por temor a lo nuevo

No me llamaba la atención

No lo necesitaba

Por otros motivos 
JA. Morales Bonilla, et al.

Tabla 1. Encuesta sobre hábitos de consumo de tabaco y alcohol (continuación).

4. ¿En tu casa fuman?

Mi pareja

Mi padre

Mi madre

Mis hermanos

Algunos/Todos

Nadie

5. ¿A qué edad empezaste a fumar?

Antes de los 13

13 a 16

17 a 20

Más de 20

6. ¿Con quién empezaste a fumar?
Sólo
Con un grupo de amigos
Con compañeros de trabajo
Con familiares
Con compañeros de estudios
Con otra gente

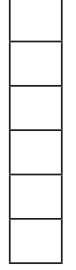

11. ¿Qué te motivó a fumar por primera vez?

Quedar bien con los amigos

Pasarlo bien

Porque así soy más hombre/mujer

Por problemas en casa

Por llevar la contraria

Por saber cómo es

Para ligar más

Otros motivos

12. ¿Cuándo fue la última vez que te fumaste un cigarrillo?

Hoy, hace un rato/horas

Ayer

La semana pasada

Durante los últimos 30 días

Más de un mes y menos de un año

Más de un año

13. ¿Te molesta el humo del tabaco?

$\mathrm{Si}$

No

14. ¿Sabes que el tabaco es cancerígeno (produce cáncer de pulmón o de laringe)?

$\mathrm{Si}$

15. ¿Te preocupa que sea cancerígeno?

$\mathrm{Si}$

No

Todos los días

Los fines de semana

Algunos días de la semana

Sólo en reuniones sociales o con los amigos

9. ¿Cuánto tiempo seguido puedes permanecer sin fumar?

Menos de un cuarto de hora

$1 / 4$ de hora -1 hora

1-2 horas

2-6 hora

Más de 6 horas

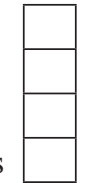

16. ¿Algún familiar tuyo ha fallecido por cáncer ocasionado por el tabaco?

$\mathrm{Si}$

No

17. Cuando fumas...

Te relajas

Ligas más

Sientes necesidad

Olvidas los problemas

Lo haces para alternar

18. ¿Sabías que el tabaco es realmente una droga que crea

En el trabajo

Con tus amigos

Cuando estás nervioso

Cuando te lo prohíben

Otras situaciones adicción?

$\mathrm{Si}$

No

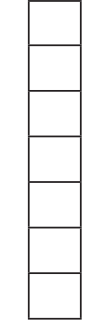

19. ¿Te consideras adicto/a al tabaco?

$\mathrm{Si}$

No 
Tabla 1. Encuesta sobre hábitos de consumo de tabaco y alcohol (continuación).

20. ¿Tomas bebidas alcohólicas?

$\mathrm{Si}$

No

21. ¿Has bebido algo en alguna ocasión? $\mathrm{Si}$

No

22. Si no bebes ¿Por qué razón no has bebido nunca? Porque sé que causa daño

Por temor a lo nuevo

No me llamaba la atención

No lo necesitaba

Por otros motivos

23. ¿Qué bebida alcohólica tomas con más frecuencia?

Vino
Cerveza
Cubatas
Whisky
Licores fuertes (ron, ginebra...)
Otras bebidas

24. ¿En tu casa consumen bebidas alcohólicas?
Mi pareja
Mi padre
Mi madre
Mis hermanos
Algunos/Todos
Nadie

25. ¿A qué edad empezaste a beber?

Antes de los 13

13 a 16

17 a 20

Más de 20

26. ¿Con quién empezaste a beber?
Sólo
Con un grupo de amigos
Con compañeros de trabajo
Con familiares
Con compañeros de estudios
Con otra gente

27. ¿Con qué frecuencia bebes?

Todos los días

Los fines de semana

Algunos días de la semana

Sólo en reuniones sociales o con los amigos

28. Cuando sales ¿Eliges preferentemente una bebida alcohólica?

$$
\begin{aligned}
& \mathrm{Si} \\
& \text { No }
\end{aligned}
$$

29. Bebes más...

Cuando estás solo

En tu centro de enseñanza

En el trabajo

Con tus amigos

Cuando estás nervioso

Cuando te lo prohíben

Otras situaciones

30. ¿Cuánto tiempo seguido puedes permanecer sin beber?

$$
\begin{aligned}
& \text { Menos de una hora } \\
& 1-3 \text { horas } \\
& 3-6 \text { horas } \\
& 6-12 \text { hora } \\
& \text { Más de } 12 \text { horas }
\end{aligned}
$$
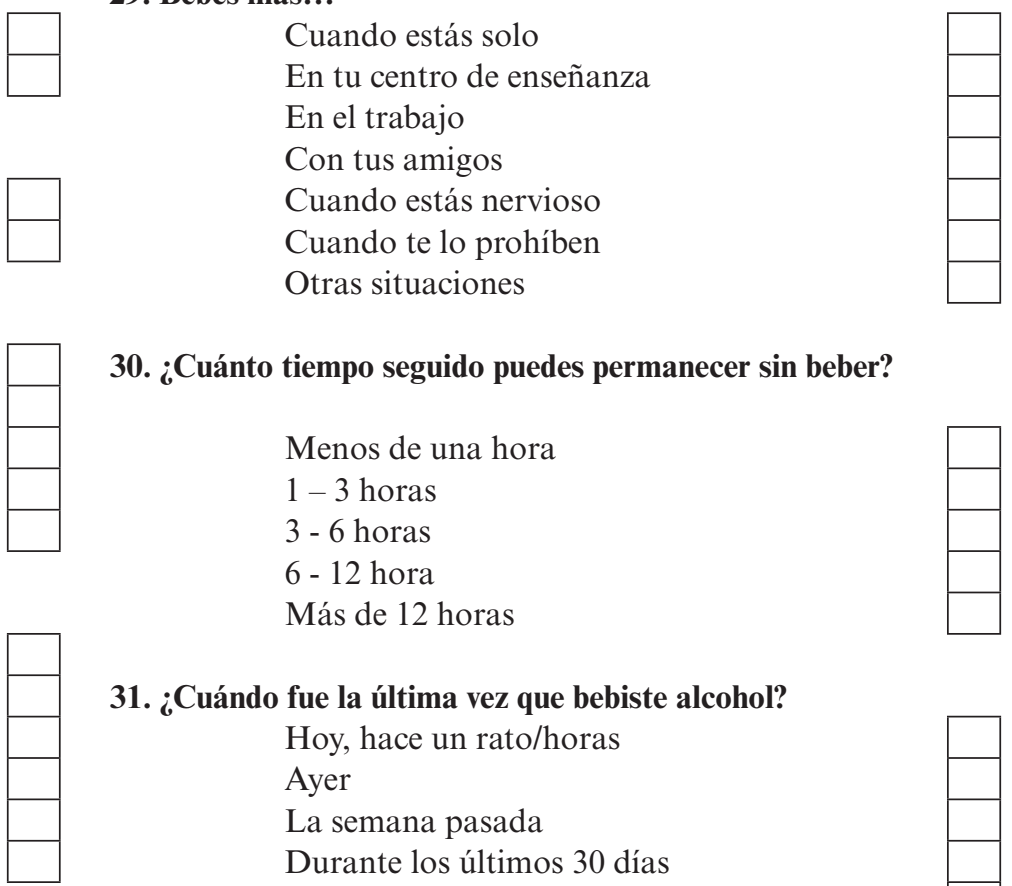

31. ¿Cuándo fue la última vez que bebiste alcohol?

Hoy, hace un rato/horas

Ayer

La semana pasada

Durante los últimos 30 días

Más de un mes y menos de un año

Hace más de un año

32. ¿Qué te llevó a beber por primera vez?

Quedar bien con los amigos

Pasarlo bien

Porque así soy más hombre/mujer

Por problemas en casa

Por llevar la contraria

Para saber cómo es

Para ligar más

Otros motivos

33. ¿Cuánto bebes aproximadamente cuando lo haces?

Una copa o cerveza

2 - 4 copas o cervezas

Más de 4 copas o cervezas
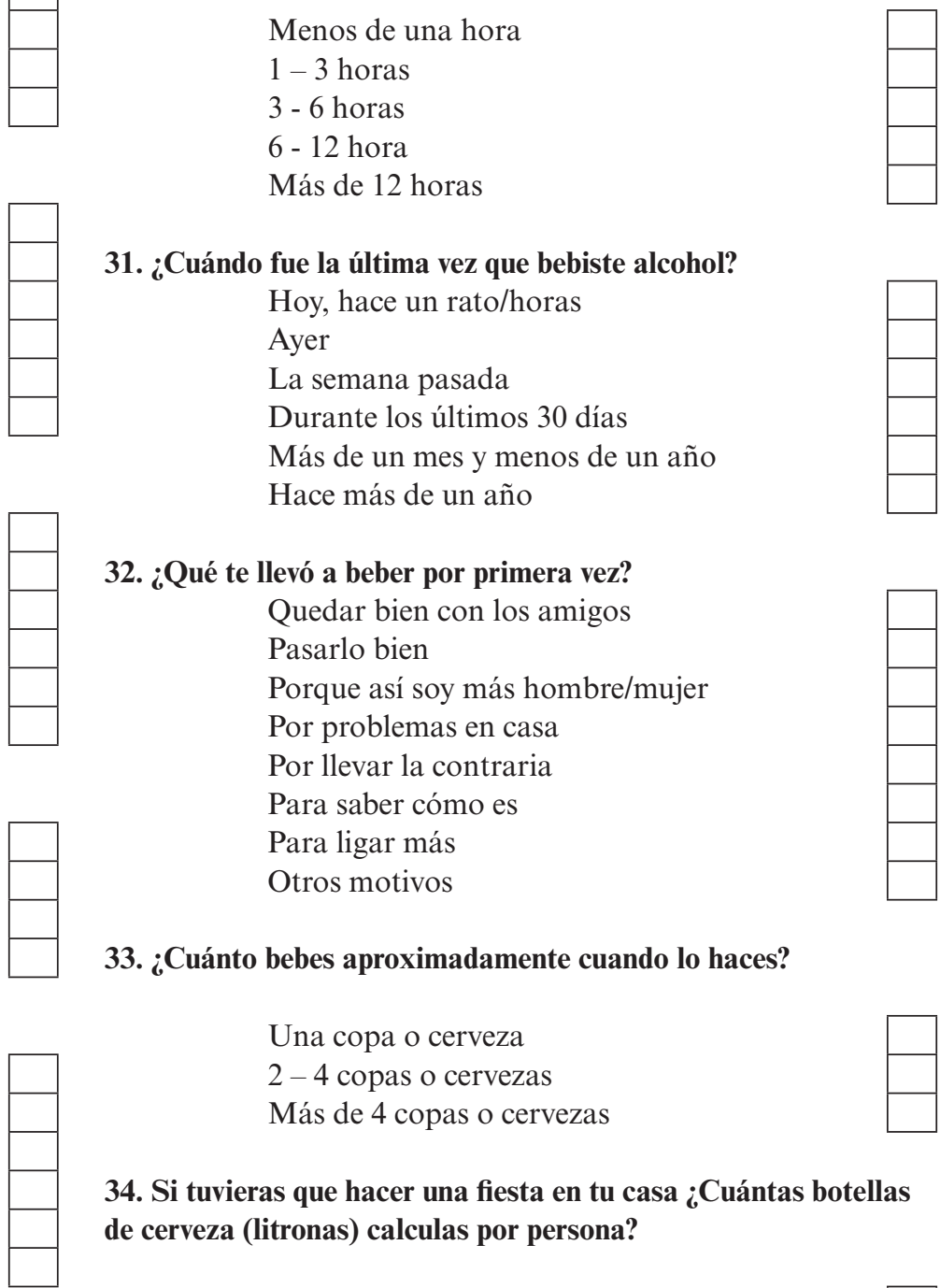

34. Si tuvieras que hacer una fiesta en tu casa ¿Cuántas botellas de cerveza (litronas) calculas por persona?

3 botellas

2 botellas

1 botella

$1 / 2$ botella

No pondría alcohol

35. ¿Conoces la graduación alcohólica de las bebidas que consumes? 
Tabla 1. Encuesta sobre hábitos de consumo de tabaco y alcohol (continuación).

36. ¿Sabes que el alcohol afecta al hígado?

$\mathrm{Si}$

No

37. ¿Algún familiar tuyo ha fallecido por problemas de hígado a causa del alcohol?

$$
\begin{aligned}
& \mathrm{Si} \\
& \mathrm{No}
\end{aligned}
$$

38. Cuando bebes...

Te relajas

Ligas más

Sientes necesidad

Olvidas los problemas

Lo haces para alternar

Te sientes más seguro de ti mismo

39. ¿Te has emborrachado alguna vez?

$\mathrm{Si}$

No

40. Después de beber ¿Te has llegado a descontrolar hasta el punto de no ser dueño de tus actos?

$$
\begin{aligned}
& \mathrm{Si} \\
& \text { No }
\end{aligned}
$$

41. ¿Alguna vez te has puesto al volante de tu coche habiendo bebido de más?

$$
\begin{aligned}
& \mathrm{Si} \\
& \mathrm{No}
\end{aligned}
$$

42. ¿Eras consciente de que no debías hacerlo?

$$
\mathrm{Si}
$$

No

43. ¿Tienes algún familiar o amigo que tenga problemas con el alcohol?

$$
\begin{aligned}
& \mathrm{Si} \\
& \text { No }
\end{aligned}
$$

44. ¿Sabías que el alcohol es realmente una droga que crea adicción?

$$
\begin{aligned}
& \mathrm{Si} \\
& \text { No }
\end{aligned}
$$

45. ¿Te consideras adicto al alcohol?

$$
\mathrm{Si}
$$

No

46. ¿Alguna vez te han dado información sobre los peligros del abuso del alcohol y del tabaco?

$\mathrm{Si}$

No

47. ¿Crees que se necesita más información sobre estos temas?

$$
\mathrm{Si}
$$

No

\section{RESULTADOS}

Se han estudiado un total de 141 individuos, el 91'5\% varones y el 8'5\% mujeres, todos ellos comprendidos entre los 18 y 24 años.

El $37^{\prime} 6 \%$ son fumadores y el $62^{\prime} 4 \%$ no, aunque de ellos, el $45^{\prime} 3 \%$ han fumado o probado el tabaco en alguna ocasión, mientras que el $54^{\prime} 7 \%$ no lo ha hecho nunca. De los que nunca han fumado o probado el tabaco, el $60 \%$, no lo hace porque no les llama la atención, y un $20 \%$ porque considera que su consumo es perjudicial para la salud.

En el $77^{\prime} 02 \%$ de las familias hay uno o más fumadores, sobre todo el padre $(36 \%)$ o la madre $(23 \%)$. Del conjunto de no fumadores, en el $48^{\prime} 86 \%$ de los casos no hay ningún fumador en su hogar.

La edad media de inicio en el consumo está entre los 13 y 16 años, $60 \%$ mujeres y.42 $86 \%$ hombres, (Figura 1) y en el primer contacto con el tabaco, el $82^{\prime} 6 \%$ lo hace a través de los amigos ( $100 \%$ de mujeres y $80^{\prime} 28 \%$ de hombres).

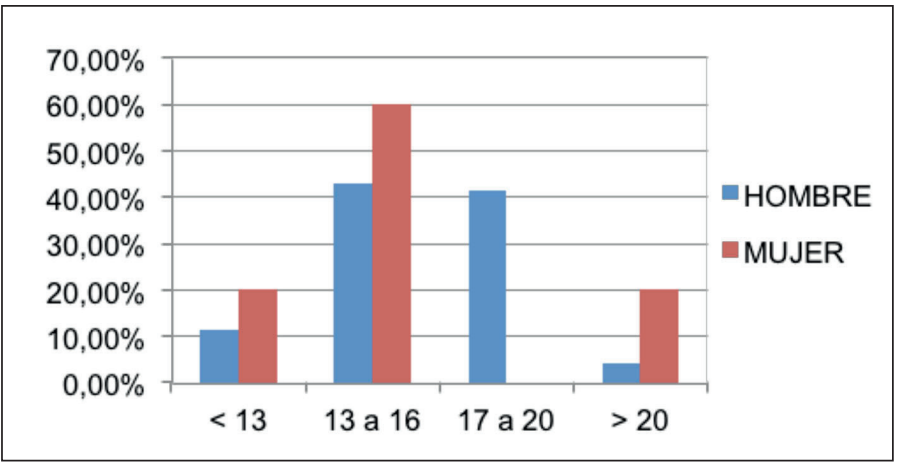

Figura 1. Edad de inicio en el consumo de tabaco.

El consumo medio es de 1 a 7 de cigarrillos al día en el $74^{\prime} 55 \%$ de los casos (Figura 2). Fuman diariamente (55'38\%), esporádicamente los fines de semana (20\%), o solo en reuniones sociales o con amigos (15'38\%). El 46'25\% de los encuestados, cuando fuma lo hace en compañía de amigos. 


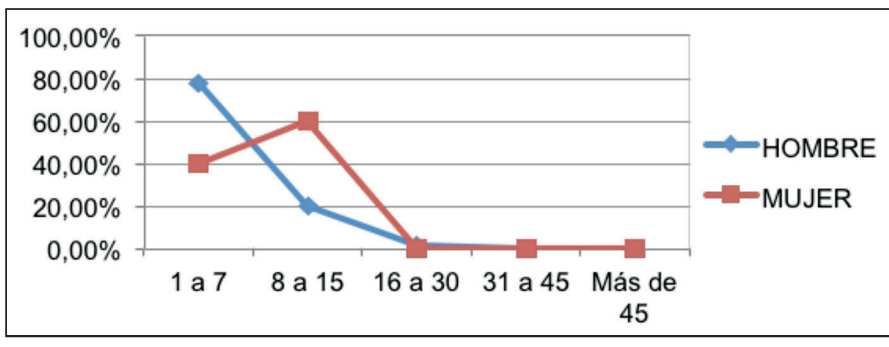

Figura 2. Consumo diario de cigarrillos.

Sobre las causas que llevaron a los encuestados a fumar por primera vez, el 74\% lo hace "por saber cómo es", el 5\% "para pasarlo bien", el 10\% "para ligar más" y el 7\% "para quedar bien ante los amigos".

El 98\% sabe que el tabaco es cancerígeno, lo que preocupa al $88 \%$, y al $18^{\prime} 44 \%$ algún familiar le ha fallecido por esta causa.

En cuanto al efecto adictivo del tabaco, el 96\% saben que produce adicción y el $21 \%$ se considera adicto y fuman sobre todo "para relajarse" $(60 \%)$, "para alternar" $(22 \%)$, "por sentir necesidad" (14\%).

Respecto al consumo de alcohol, el 82\% declaran tomar bebidas alcohólicas. De los que no beben, el 13\% no lo han probado nunca, mientras que el $87 \%$ si lo han hecho en alguna ocasión. Los motivos que alegan los que no han probado nunca el alcohol son, en el mismo porcentaje (33'33\%), "porque es dañino para la salud", "porque no les llama la atención" o "porque no lo necesitan".

Las bebidas alcohólicas que toman más frecuentemente está la cerveza $\left(46^{\circ} \%\right)$, cubatas $(22 \%)$, licores fuertes $\left(13^{\prime} 72 \%\right)$ y vino $\left(5^{\prime} 75 \%\right)$. Conocen la graduación de las bebidas el $85 \%$ de los encuestados.

En el entorno familiar, en el $72 \%$ de los casos se produce consumo de alcohol, predominando el consumo del padre (31\%).

La edad media de inicio de consumo se sitúa entre los 13 y 16 años (Figura 3), en el 54\% de los encuestados, siendo el factor inductor al consumo los amigos en el $80^{\prime} 5 \%$ de las veces, o la influencia de familiares $\left(6^{\prime} 5 \%\right)$.

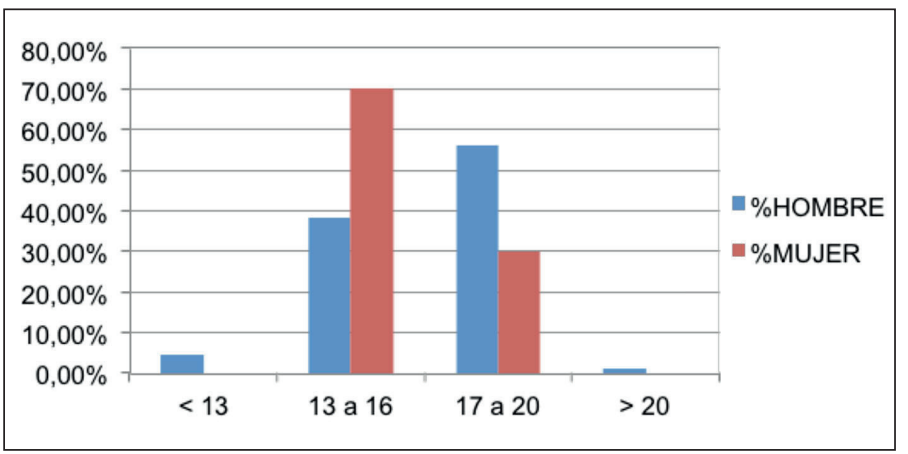

Figura 3. Edad de inicio en el consumo de alcohol.

El consumo predomina los fines de semana en el $49^{\prime} 5 \%$ y se hace con motivo de reuniones sociales en el $44 \%$ de las veces. El 81 '3 de los casos bebe más cuando está en compañía de amigos. Cuando alternan, el 56'83\% eligen el alcohol, de los que el

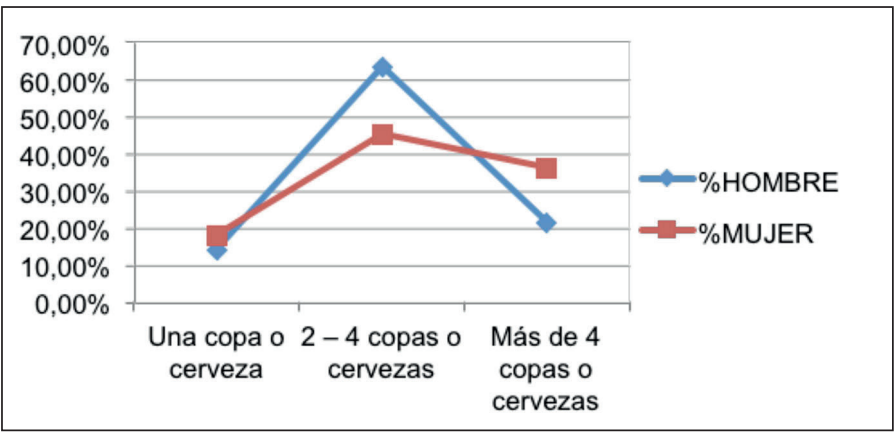

Figura 4. Cantidad de alcohol consumida cuando beben.

62 ' $3 \%$ toma entre 2 y 4 copas o cervezas, el $23 \%$ toma más de 4 y el 14'62\% toma sólo una (Figura 4).

La primera vez que bebieron, el 46'34\% lo hizo "para saber cómo era", el 31'71\%, "para pasarlo bien", 2'44\% "para ligar" y el 1'22\% "para quedar bien con los amigos" o "para demostrar su hombría".

El 98\% saben que el alcohol produce daño hepático, el 12\% han tenido algún familiar fallecido por esta causa y el $23 \%$ tienen familiares o amigos que en la actualidad tienen problemas con el alcohol. Aun así, el 56\% bebe "para alternar", el 21'5\% "para evadirse de los problemas", el 13\% "para relajarse", el 11\% "para sentirse más seguro de sí mismo" y el 8’4\% "para ligar".

El $81{ }^{\prime} 5 \%$ reconoce haberse emborrachado alguna vez y el $26^{\prime} 24 \%$ ha bebido en exceso, hasta llegar a no ser dueños de sus actos. El 22\% ha realizado conductas de riesgo tras la ingesta de alcohol, poniéndose al volante de un vehículo el 23\% hombres y el 17\% de las mujeres. El 93\% de todos ellos eran conscientes de que no debían hacerlo y aun así lo hicieron.

El 95'5\% de los encuestados reconocen saber los efectos adictivos del alcohol y sólo el 0'71\% se considera adicto a él. El 93,62\% dice haber sido informado sobre los peligros del abuso de alcohol y tabaco, y el 68,09\% considera que esta información es insuficiente.

\section{DISCUSIÓN}

La técnica de muestreo utilizada selecciona a ciertos individuos dentro de un determinado colectivo. Aunque en principio pueda parecer que exista un sesgo de selección por haber elegido a un grupo en concreto, los resultados pueden ser pertinentes y son bastante representativos de la población general por tratarse de un grupo de jóvenes de 18 a 24 años, que provienen de toda la geografía nacional y que se diferencian poco de otros jóvenes de la misma edad a los que hubiéramos entrevistado en otro momento y lugar, como se puede comprobar si se compara con otros resultados, como el realizado en Castilla La Mancha ${ }^{1}$. Sí puede ser que haya algún sesgo en el estudio comparativo entre mujeres y hombres por tratarse de un grupo militar donde hay 129 hombres y sólo 12 mujeres. Obviando estas comparaciones, el estudio presenta buena fiabilidad o precisión, en el sentido de que las mediciones repetitivas de las cuestiones propuestas nos darían las mismas tendencias. Por otro lado también podemos afirmar que existe bastante exactitud o validez, ya que los datos 


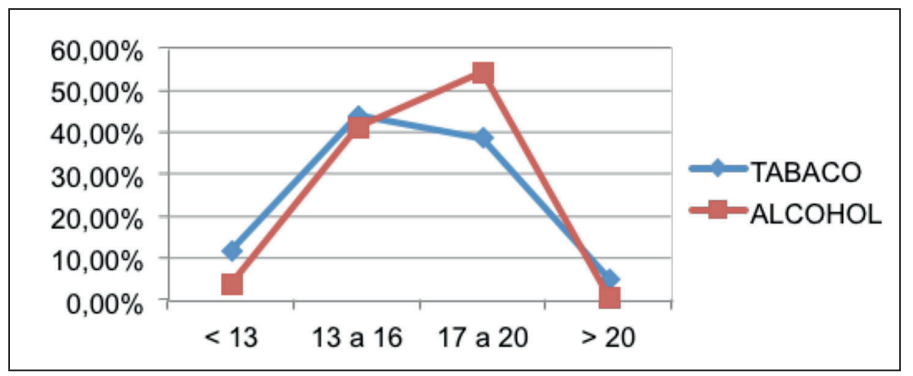

Figura 5. Edad de inicio en el consumo de tabaco y alcohol.

encontrados se corresponden con las tendencias en el consumo que se determinan en la última Encuesta Nacional de Salud (ENSE) publicada 5 .

Estamos ante dos drogas muy arraigadas en las costumbres sociales y con un consumo muy amplio en todos los sectores de la población. En ambas se produce un inicio precoz, antes de los 16 años (Figura 5), al igual que ocurre en la ENSE 20065 , y en EDADES $2011^{6}$ y entre los 16 y los 20 años se inicia una escalada hacia un mayor consumo, sobre todo en el caso del alcohol. Como también concluyó Cortés Tomás en su estudio ${ }^{16}$, los encuestados beben más los fines de semana o con ocasión de reuniones sociales y si están acompañados de sus amigos y, cuando alternan, eligen preferentemente bebidas con alcohol, tomando mayoritariamente de 2 a 4 copas, si no más. En el caso del tabaco, ocurre algo parecido. El consumo de tabaco no se reduce a los fines de semana, sino que es diario, pero sobre todo han empezado a fumar con los amigos y fuman más si están con ellos. Esto nos lleva a hacernos una idea del arraigo social y la permisividad de estas dos drogas legales, como también se observa en el estudio de la Universidad de Zaragoza ${ }^{17}$. El estudio también refleja que la familia tiene gran influencia en los hábitos de consumo, al igual que observa RuizRisueño ${ }^{18}$. Es curioso ver cómo, en algunos casos, el inicio en el consumo de alcohol se produce en casa.

Aunque el hecho de que el tabaco es adictivo y cancerígeno es conocido casi por el total de los encuestados y además les preocupa este dato, siguen fumando "para alternar", "porque se relajan", e incluso "porque sienten necesidad", como ocurre a grandes rasgos en el estudio de Pérez Milena ${ }^{19}$, pero el 79,43\% no se considera adicto al tabaco. En cuanto a la bebida, saben que produce daño hepático, e incluso pueden tener familiares actualmente con problemas por el alcohol o ya fallecidos por esta causa, pero beben "para alternar", "para olvidar problemas" o "porque les relaja”, como ya comprobó López García también en su estudio ${ }^{20}$.

También es bastante preocupante el dato asociado al alcohol de que un $21,99 \%$ se han puesto al volante de un coche, como en el estudio realizado por Calafat et $\mathrm{al}^{21}$, y prácticamente todos sabían además que no debían hacerlo.

Ahora que conocemos estos datos, podemos planear estrategias de prevención, objetivo también de este estudio. Estas no deben limitarse a la mera información, sino que deben de integrar una serie de actividades dirigidas a adoptar estilos de vida más saludables y ofrecerles otras alternativas de ocio. La prevención ha de ser, si cabe, aún mayor en el medio donde se realiza este estudio debido a su futuro desempeño profesional como de Agentes de la Autoridad. Al finalizar su periodo forma- tivo tendrán que realizar funciones policiales, e incluso controlar y prevenir del consumo de alcohol y drogas, relacionados con la seguridad vial y ciudadana, por lo que debemos formar personas mucho más concienciadas con los problemas que se derivan de estos consumos.

Las importantes limitaciones que tiene este trabajo asociadas sobre todo con el tamaño de la muestra y la selección de conveniencia, convierten al estudio en válido sólo para los sujetos participantes, que en definitiva son nuestro objetivo en este momento.

\section{CONCLUSIONES}

Las tendencias de consumo de alcohol y tabaco en este colectivo se corresponden, en rangos de edad y sexo, con las de la población general.

Más del 90\% de los alumnos tienen conocimiento de las nocivas consecuencias del consumo y abuso del alcohol y el tabaco pero un $20 \%$ de los encuestados ha realizado actividades de riesgo bajo les efectos del alcohol.

Debido a su rol de agentes de la autoridad en periodo de formación, deben implementarse medidas que ayuden a formar personas mucho más concienciadas con los problemas derivados de estos consumos.

\section{BIBLIOGRAFÍA}

1. Martínez Lorca M, Alonso Sanz C, Montañés Rodríguez J. Consumo de tabaco, alcohol y cannabis en adolescentes Castellano-Manchegos. Rev Esp Drogodep 2010; 35(1): 78-91.

2. Organización Mundial de la Salud. Informe OMS sobre la epidemia mundial de tabaquismo. Informe de un grupo científico de la OMS. Ginebra: OMS; 2009.

3. Solano Mendoza P, García García V, Bascones Martínez A. ¿Existe suficiente evidencia en la literatura para considerar el tabaco y el alcohol como principales factores de riesgo del cáncer de lengua? Av Odontoestomatol 2010; 26(1): 31-44.

4. Estrategia para la prevención y el control de las enfermedades no transmisibles, 2012-2015. 28 ${ }^{\text {a }}$ Conferencia Sanitaria Panamericana; 2012 Septiembre 17-21; Washington DC, Estados Unidos: Organización Panamericana de la Salud.

5. Ministerio de Sanidad, Servicios Sociales e Igualdad. Presentación de la Encuesta Nacional de Salud: ENSE 2006-07 [Internet]; 2008. [Acceso 2/01/2012]. Disponible en: http://www.msssi.gob.es/estadEstudios/estadisticas/encuestaNacional/encuestaNac2006/EstilosVidaPorcentaje.pdf

6. Plan Nacional sobre Drogas. Encuesta Domiciliaria sobre Alcohol y Drogas en España (EDADES). Madrid: Ministerio de Sanidad, Política Social e Igualdad, 2011. Disponible en: http://www.pnsd.msc.es/Categoria2/observa/ pdf/oed2011.pdf

7. Delegación del gobierno para el plan nacional sobre drogas. Encuesta domiciliaria sobre drogas en España, 2009-2010. Ministerio de Sanidad, Política Social e Igualdad; 2010.

8. Observatorio para la Prevención del Tabaquismo. Informe a las Cortes Generales de evaluación del impacto sobre la salud pública de la Ley 42/2010. Ministerio de Sanidad, Política Social e Igualdad, 2013.

9. O.M.S. Alcohol. [Internet] Nota descriptiva 349. Febrero de 2011 [Acceso 28 de octubre de 2013]. Disponible en: http://www.who.int/mediacentre/factsheets/fs349/es/

10. Zaldíbar Basurto F, Lopez Rios F, Garcia Montes JM, Molina Moreno A. Consumo autoinformado de alcohol y otras drogas en población universitaria española. Electronic Journal of Research in Educational Psychology 2011; 9(1): 113-132. 


\section{Consumo de alcohol y tabaco entre los alumnos de un centro de formación de Fuerzas y Cuerpos de...}

11. Cassiani CA, Cubides AM, Borrero Varona MT, Marimón Trespalacios W. Alcohol y trauma: Un problema prioritario de salud pública. Salud Uninorte. Barranquilla (Col) 2012; 28(1): 131-149.

12. Acevedo González A, Rech Mertínez A, Rodriguez Roig A. Incidencia del alcoholismo según características sociales. Comunidad Policlínico "Pedro Fonseca”. La Lisa. Años 2011-2012. Revista Cubana de Tecnología de la Salud [revista en internet] 2013 [acceso 31 de octubre de 2013]; 3(2). Disponible en: http://www.revtecnologia.sld.cu/indez.php/tec/article/viewArticle/63

13. Revista Tráfico y Seguridad Vial, de la Dirección General de Tráfico, $\mathrm{n}^{\circ} 210$ de Oct. 2011, p. 44-45.

14. Sáenz J. Alcohol y medicamentos en el trabajo. Revista de la Confederación de Empresarios Baleares (CAEB), 2000. Revista virtual.[Internet] [acceso 30 dic 2011]. Disponible en: www.caeb.es/embal/nr23/ead_5.htm

15. Europa Press. El alcohol, causa de siniestralidad laboral.[Internet] [acceso 28 Dic. 2011]. Disponible en: http://www.lukor.com/hogarysalud/04121702.htm

16. Cortés Tomás M T, Espejo Tort B, Martín del Río B, Gómez Iñíguez C. Tipologías de consumidores de alcohol dentro de la práctica del botellón en tres ciudades españolas. Psicothema. 2010; 22 (3): 363-8
17. Gómez Bahillo C, Ezquerro Villarroya E. El botellón entre los jóvenes de la ciudad de Zaragoza. Análisis y propuestas alternativas. Zaragoza: Universidad de Zaragoza y Ayuntamiento de Zaragoza; 2012

18. Ruiz-Risueño Abad J, Ruiz-Juan F, Zamarripa Rivera JI. Alcohol y tabaco en adolescentes españoles y mexicanos y su relación con la actividad físicodeportiva y la familia. Rev Panam Salud Pública. 2012; 31(3):211-20.

19. Pérez Milena A, Martínez Fernández ML, Redondo Olmedilla M, Álvarez Nieto C, Jimenez Pulido I, Mesa Gallardo I. Motivaciones para el consumo de tabaco entre los adolescentes de un instituto urbano. Gac Sanit. 2012; 26(1): 51-7.

20. López García KS, Esparza Almanza SE, Guzmán Facundo FR, Alonso Castillo MM, Rodríguez Aguilar L. Expectativas del consumo de alcohol y tabaco en adolescentes escolares. Revista Cientifico Electrónica de Psicología. Revista virtual [Internet] 2009: No 7 [Acceso 22/10/2013]. Disponible en: http://dgsa.uaeh.edu.mx/revistas/index.php/psicologia/article/ view/118/109

21. Calafat A et al. Alcoholemias en contextos recreativos nocturnos como sistema de prevención. Actas Esp Psiquiatr 2013; 41(1):10-6. 